\title{
Does the Human Dorsal Stream Really Process a Category for Tools?
}

\author{
Satoshi Sakuraba, ${ }^{1}$ Shinya Sakai, ${ }^{2}$ Masanori Yamanaka, ${ }^{2}$ Koichi Yokosawa, ${ }^{2}$ and Kazumi Hirayama ${ }^{3}$ \\ ${ }^{1}$ Graduate School of Health Sciences and ${ }^{2}$ Faculty of Health Sciences, Hokkaido University, Sapporo 060-0812, Japan, and ${ }^{3}$ Yamagata Prefectural University \\ of Health Sciences, Yamagata 990-2212, Japan
}

Previously, Almeida et al. (2008) used a technique called Continuous Flash Suppression to show that human dorsal stream cortical areas specifically responded to a "tool category." Here, we used the same technique to clarify what attributes of tools are processed in the dorsal stream. We examined surface attributes and shape. A significant priming effect was found when we removed surface attributes by using line drawings instead of photographs. In a second experiment, we manipulated shape and we found that there were no significant priming effects when we used nonelongated tool pictures as tool prime stimuli. To better clarify the effect of shape attributes on priming effects, we conducted a further experiment using elongated stick-like rectangles as prime stimuli and found that elongated shapes significantly shortened the reaction time to the tool pictures as target stimuli. Additionally, when elongated vegetables were used as prime stimuli, the reaction time to the tool pictures as target stimuli was also significantly shortened, but there was no effect when stubby vegetables were used. Finally, when we controlled for orientation by presenting rotated elongated stick-like rectangles, diamond shapes, and cut circles as prime stimuli, we found that rectangles replicated the same significant priming effect as previous experiments, but the others did not. These results suggest that the dorsal stream processes elongated shapes but does not process the tool category specifically.

\section{Introduction}

It has been suggested that the human brain has two pathways or streams to process visual information (Goodale and Milner, 1992; Rizzolatti and Matelli, 2003). The dorsal stream arises in early visual cortex in the occipital lobe and projects to the posterior parietal cortex. Activity within the dorsal stream has been tied to reaching and grasping based on online processing with respect to motion, spatial location, shape, and orientation of objects. Patients with dorsal stream damage have trouble reaching for or grasping objects, but can verbally describe the object's attributes (Goodale, 2011). In contrast, the ventral stream, which also arises in early visual cortex in the occipital lobe but projects to the inferotemporal cortex, is thought to analyze the color and shape of objects and associate their features with meanings. $\mathrm{Pa}$ tients with ventral stream damage have been reported to have trouble recognizing the size, shape, and orientation of objects, but can reach and grasp objects precisely (Goodale et al., 1991).

Recent reports have suggested that the dorsal stream may process categorical information rather than simply reflecting spatial or motion attributes. Several studies have used fMRI to explore potential relationships between dorsal stream activity and items in the "tool" category, though there are many studies indicating

Received Aug. 3, 2011; revised Jan. 24, 2012; accepted Jan. 27, 2012

Author contributions: S. Sakuraba, S. Sakai, M.Y., and K.H. designed research; S. Sakuraba performed research; S. Sakuraba and S. Sakai contributed unpublished reagents/analytic tools; S. Sakuraba, S. Sakai, K.Y., and K.H. analyzed data; S. Sakuraba, S. Sakai, M.Y., K.Y., and K.H. wrote the paper.

The authors declare no competing financial interests.

Correspondence should be addressed to Shinya Sakai, Faculty of Health Sciences, Hokkaido University, N-12 W-5 Kita-ku, Sapporo 060-0812, Japan. E-mail: saka@@h.hokudai.ac.jp.

DOI:10.1523/JNEUROSCI.3973-11.2012

Copyright $\odot 2012$ the authors $\quad 0270-6474 / 12 / 323949-05 \$ 15.00 / 0$ that a part of ventral visual stream (the left middle temporal gyrus and adjacent areas) is activated by tools or tool-related actions (Chao et al., 1999; Beauchamp et al., 2002, 2003; Valyear and Culham, 2010; Bracci et al., 2011). Chao and Martin (2000) showed that the dorsal stream was activated during the recognition and identification of tools. Vingerhoets et al. (2009) also suggested that use-responsive contrasts revealed a superior parietal region. Mahon et al. (2010) demonstrated that the posterior superior parietal lobule (SPL) and inferior parietal lobule showed differential BOLD responses for tool stimuli compared with nontool stimuli. SPL receives visual input from cortical area V6, which belongs to the main dorsal extrastriate visual pathway and appears to be a purely visual area (Colby et al., 1988; Rizzolatti and Matelli, 2003). Moreover, Almeida et al. $(2008,2010)$ used a visual masking technique called Continuous Flash Suppression (CFS), a method introduced by Tsuchiya and Koch (2005), to examine unconscious visual information processing using interocular suppression and found significant priming effects for tools but not animals. Fang and He (2005) suggested that the dorsal stream processes priming stimuli, as demonstrated with fMRI experiments where the dorsal stream was activated preferentially when a dynamic $(10 \mathrm{~Hz})$ random-noise pattern was presented to the dominant eye and a static-tool prime stimulus was presented to the nondominant eye for $200 \mathrm{~ms}$ while subjects wore red/green anaglyph glasses. Together, these results have been interpreted to suggest that the dorsal stream processes tools as a category.

However, it is unlikely that the dorsal stream could discriminate tools from other objects in the early stages of visual processing. Lin and $\mathrm{He}$ (2009) suggested that, to determine whether tools are preferentially represented in the dorsal stream, it is important to characterize the levels of representation for tools. Almeida et al. (2008) also 
noted that the tool stimuli they used all had an elongated principal axis and this feature might have impacted the results. Does the human dorsal stream really process a category for tools as Almeida et al. (2008) noted? Otherwise, are some common visual attributes of the tools (i.e., surface feature, shape, and orientation) merely processed? In this study, we conducted five serial experiments with CFS to solve this research question.

\section{Materials and Methods}

Participants. Eighteen (Experiment 1) and 20 (Experiments 2-5) graduates and undergraduate students participated in our experiments (Experiment 1: five females and 13 males, age range $20-25$ years, mean age $23.1 \pm 1.6$; Experiment 2: eight females and 12 males, mean age $22.4 \pm 2.7$; Experiments 3 and 4 : six females and 14 males, mean age 22.1 \pm 2.0 ; and Experiment 5: seven females and 13 males, mean age $22.3 \pm 1.2$ ). All subjects had normal vision and were naive as to the experimental hypotheses. All subjects gave written informed consent.

Apparatus. All experiments were run on an IBM ThinkPad G41 2881-B4J. Stimuli were presented on a 22-inch monitor (TOTOKU CV921, Totoku Electric) at a resolution of $1280 \times 1024$ pixels and with a refresh rate of $100 \mathrm{~Hz}$. Stimulus presentation was controlled by DMDX (Foster and Foster, 2003).

Stimuli. Stimuli were created according to the method of Almeida et al. (2008; Figure 1). The viewing distance was $150 \mathrm{~cm}$. For each category, we prepared five images and one image was selected as the prime stimulus. The remaining four images in each category were used as target stimuli. In Experiment 1, we used line drawings as prime and target stimuli. In Experiment 2, we used tool pictures without elongated shape components, namely stubby tools (e.g., a punch, a squeezer, a mouse, and so on). The tool pictures in noncanonical view were used as previous tool prime stimuli. In Experiment 3, elongated stick figures were used as prime stimuli. In Experiment 4, elongated and stubby vegetable pictures were presented as prime stimuli. Because the elongated shapes involve an orientation component, we could not exclude the possibility that orientation, rather than shape attribute, explained the results. Therefore, we conducted Experiment 5 to clarify this. We used elongated stick figures, diamond shapes, and cut circles that were rotated in $45^{\circ}$ increments as prime stimuli. These geometric figures were presented in random order with equal frequency in their types and angles. For target stimuli, we used the same stimuli as our previous experiments so that the angles of prime and target stimuli were identical at a rate of $25 \%$. Examples of these images are presented in Figure 2. For all experiments, stimuli subtended $\sim 7^{\circ}$ of visual angle and $70 \%$ noise were added to the target stimuli.

Procedure. Before starting experiments, we identified each subject's dominant eye. Subjects viewed a filled circle centered in the monitor through a circular cylinder and were asked to close each eye alternately. The subjects verbally reported when the filled circle looked similar to the binocular viewing condition, and this was defined to be the dominant eye. Before each experiment, we conducted a task to confirm subjects' blindness to the prime stimuli (Table 1). In this task, we randomly presented random-noise patterns with or without prime pictures at the same rate, and subjects were asked to indicate by pressing a key whether or not they detected something other than the noise patterns. If subjects could not really see the prime stimuli, percentage correct performance would be close to $50 \%$ (chance level). We adopted the contrast level that produced chance detection performance for the subsequent experiments. Table 1 summarizes the results of this task.

In our experiments, subjects were asked to answer the category of presented targets (e.g., tools or animals) by pressing one of two keys. Each trial started with the presentation of a fixation cross $(500 \mathrm{~ms})$.

\section{A 0 degree}
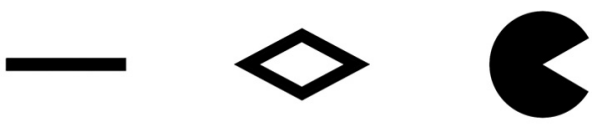

B 135 degree
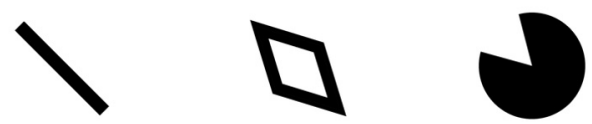

Figure 2. The images used in Experiment 5. We used elongated stick figures as in Experiment 2 (left), diamond shapes (center), and cut circles (right). These three images presented in this experiment are rotated in $45^{\circ}$ steps. For example, there are $0^{\circ}$ images $(\boldsymbol{A})$ and $135^{\circ}$ rotated images (B).

Table 1. Experimental measures of prime stimuli invisibility

\begin{tabular}{lllllll}
\hline & \multicolumn{6}{c}{ Experiment Experiment Experiment Experiment Experiment } \\
& Photo 1 & 2 & 3 & 4 & 5 \\
\hline$n$ & 18 & 18 & 20 & 20 & 20 & 20 \\
Mean error rate, \% & 50.94 & 50.65 & 51.26 & 50.60 & 49.57 & 52.15 \\
SD & 4.30 & 3.39 & 3.09 & 5.01 & 3.63 & 4.28 \\
SEM & 1.02 & 0.76 & 0.69 & 1.12 & 0.76 & 0.96 \\
Maximum error rate, \% & 62 & 62 & 58 & 62 & 58 & 62 \\
Minimum error rate, \% & 45 & 45 & 45 & 45 & 45 & 45 \\
\hline
\end{tabular}

$n$, number of subjects.

Subsequently, $10 \mathrm{~Hz}$ dynamic high-contrast random-noise patterns and low-luminance low-contrast prime stimuli were presented to the dominant and nondominant eyes, respectively, for $200 \mathrm{~ms}$. Finally, a target stimulus was presented until the subject responded, or for a maximum of $3 \mathrm{~s}$. The trial set of 16 stimuli was repeated 10 times. After five blocks, the keys representing each category were switched. All subjects performed a practice set of trials before the experiment.

Analyses. Tool and animal priming effects were calculated by subtracting reaction times (RTs) in the congruent condition (i.e., coincidence of category in prime and target stimuli) from RTs in the incongruent condition. In Experiment 1 and 2, the tool priming effect was calculated by subtracting "mean RTs when animal prime stimuli were presented" from 


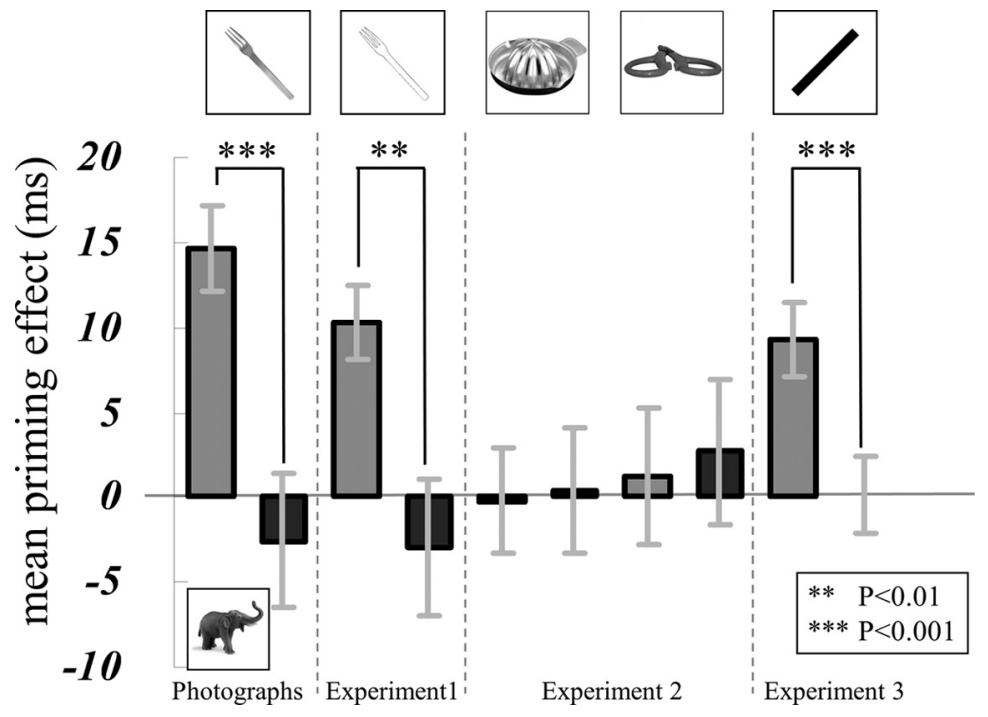

Figure 3. Priming effects in Experiments 1, 2, and 3. Light and dark gray bars represent mean priming effects to tool targets and animal targets, respectively. Error bars indicate SEM. The pictures represent examples of the prime stimuli we used.

\begin{abstract}
"mean RTs when tool prime stimuli were presented." Likewise, the animal priming effect was calculated by subtracting "mean RTs when tool prime stimuli were presented" from "mean RTs when animal prime stimuli were presented." In Experiments 3 and 5, we calculated the two priming effects replacing "mean RTs when tool prime stimuli were presented" by "mean RTs when geometric shape prime stimuli were presented." In Experiment 4, we calculated the tool priming effect by subtracting "mean RTs when stubby vegetable pictures were presented" from "mean RTs when elongated vegetable pictures were presented." The animal priming effect was calculated by subtracting "mean RTs when elongated vegetable pictures were presented" from "mean RTs when stubby vegetable pictures were presented." A paired $t$ test was performed on the mean priming effects at the $5 \%$ significance level using PASW Statistics 18.0 (SPSS). If subjects pressed the incorrect key, the RT data were excluded, and the following trial was also excluded because RTs immediately after an error tended to be prolonged.
\end{abstract}

\section{Results}

We first confirmed that priming stimuli were invisible to the subjects before each experiment (summarized in Table 1). We then replicated Almeida et al. (2008), whose analyses of priming effects showed that mean priming effects for tools $(14.6 \mathrm{~ms}$, $\mathrm{SEM}=2.52)$ and animals $(-2.6 \mathrm{~ms}, \mathrm{SEM}=3.94)$ differed significantly $\left(t_{(17)}=4.032, p<0.001\right.$; Fig. 3$)$.

In the present Experiment 1, we used line drawings as prime and target stimuli. Similar to Almeida et al. (2008), significantly greater priming effects were seen for tools than for animals (10.30 $\mathrm{ms}$, SEM 2.19; and $-3.0 \mathrm{~ms}, \mathrm{SEM}=4.06$, respectively; $t_{(17)}=$ $3.519, p<0.01)$. Surface attributes such as texture and color were not necessary to achieve the greater priming for tool stimuli.

In Experiment 2, we aimed to clarify whether the significant priming effects in Almeida et al. (2008) and our previous study were due to the representation of a tool category (as Almeida et al. noted) or to the elongated shapes common in tools. We found tools without elongated shapes and used these tools as prime stimuli. In contrast with the previous experiment, there were no significant differences between tool and animal priming effects. Mean priming effects were $-0.24 \mathrm{~ms}(\mathrm{SEM}=3.12)$ for tools and $0.35 \mathrm{~ms}(\mathrm{SEM}=3.72)$ for animals $\left(t_{(19)}=-0.156, p=0.877\right)$. Similarly, when we presented tools in noncanonical views as tool prime stimuli, there were also no significant differences between tool and animal priming effects. Mean priming effects were 1.21
$\mathrm{ms}(\mathrm{SEM}=3.97)$ for tools and $2.66 \mathrm{~ms}$ $(\mathrm{SEM}=4.22)$ for animals $\left(t_{(19)}=-0.291\right.$, $p=0.774)$.

To determine more clearly whether the priming was due to shape or category, Experiment 3 used elongated rectangles as prime stimuli, and significant differences in priming were found between the tool and animal categories. Mean priming effects were $9.26 \mathrm{~ms}(\mathrm{SEM}=2.15)$ for tools and $0.12 \mathrm{~ms}(\mathrm{SEM}=2.30)$ for animals $\left(t_{(19)}=4.844, p<0.001\right)$.

Because elongated stick-like prime stimuli showed a significant priming effect for tools, Experiment 4 was designed to ask whether the relevant attribute was the elongated shape. When elongated vegetables were used as prime stimuli, significant differences were seen between tools and animals, consistent with the previous experiments. Mean priming effects were $9.68 \mathrm{~ms}(\mathrm{SEM}=3.31)$ for tools and -2.10 $\mathrm{ms}(\mathrm{SEM}=2.55)$ for animals $\left(t_{(19)}=\right.$ $3.577, p<0.01$ ). However, when stubby vegetables were shown instead of elongated ones, no significant difference in priming effects were found (tools: $-0.47 \mathrm{~ms}, \mathrm{SEM}=3.09$; animals: 0.52 $\left.\mathrm{ms}, \mathrm{SEM}=2.72 ; t_{(19)}=-0.272, p=0.789\right)$. This suggests that the priming effects in Experiment 3 were due to the elongated shape attribute rather than the category.

Finally, we conducted Experiment 5 to ask whether the priming effect was due to the orientation of the presented images. Consistent with the previous experiments, significant differences were seen between tools and animals only when elongated stick figures were used as prime stimuli (Fig. 4). Mean priming effects were $9.40 \mathrm{~ms}(\mathrm{SEM}=3.19)$ for tools and $-5.01 \mathrm{~ms}(\mathrm{SEM}=3.17)$ for animals $\left(t_{(19)}=2.716, p=0.014\right)$. On the other hand, when diamond shapes and cut circles were shown as prime stimuli, no significant difference in priming effects were found. For diamond shapes, mean priming effects were $0.323 \mathrm{~ms}(\mathrm{SEM}=2.64)$ for tools and $-2.87 \mathrm{~ms}$ (SEM 3.80) for animals $\left(t_{(19)}=0.579, p=\right.$ $0.570)$. Moreover, for cut circles, mean priming effects were $-3.35 \mathrm{~ms}(\mathrm{SEM}=4.99)$ for tools and $-6.19 \mathrm{~ms}$ (SEM 3.84) for animals $\left(t_{(19)}=0.347, p=0.732\right)$.

\section{Discussion}

In this study, we aimed to reveal whether the human dorsal stream processes the tool category or some common visual attributes of the tools (surface feature, shape, and orientation). We used a CFS technique that elicited priming, consistent with previous reports that the dorsal stream processes unconscious visual information (Goodale and Milner, 2004).

Like Almeida et al. (2008, 2010), we found that RTs were significantly shortened when tool target stimuli were presented following tool prime stimuli, validating our methods. In Experiment 1 , we found similar priming effects using line drawings of tools that removed texture and color from the priming stimuli. Because CFS relies on red/green anaglyphs, we cannot fully address the effects of color attributes. However, the significant priming effect between line drawings of tools and animals suggests that color information may not be very important in the dorsal stream. Experiment 1 indicated that information about tool stimuli could be processed in the dorsal stream even when no surface attributes (i.e., textures and colors) were present. Cavina- 
Pratesi et al. (2010) showed that the anterior collateral sulcus and lingual gyrus process colors and that the posterior collateral sulcus processes textures. The collateral sulcus is part of the ventral stream, so our results are consistent with these findings.

In Experiment 2, we aimed to clarify the effect of shape attributes of tools. Almeida et al. (2008) noted that their tool stimuli had an elongated principal axis. Therefore, it was possible that their results were due to the elongation rather than category. If significant priming effects were found with stubby tools for prime stimuli, we could suggest that the dorsal stream processes the tool category as Almeida et al. (2008) indicated. Otherwise, their findings could be interpreted as the dorsal stream carrying elongated shape attributes. To investigate this, we found tool pictures without elongated shape components for tool prime stimuli (i.e., stubby tool pictures and the tool pictures in noncanonical view). These experiments showed no significant differences between tool and animal priming effects and indicated that the dorsal stream processes shape attributes rather than category of tools. However, there are many differences between elongated and stubby tools. Many elongated tools are used by grasping their handles, while stubby tools are generally used where they are placed on flat surfaces (e.g., a desk). In addition, the tool pictures in noncanonical views were more difficult to recognize than tool pictures in canonical view. Therefore, we conducted further experiments to clarify the relationships between shape attributes and the dorsal stream.

To confirm the possibility that the dorsal stream processes shape attributes more clearly, we used elongated stick-like rectangles as prime stimuli (Experiment 3). As we predicted, these prime stimuli significantly shortened RTs to tool pictures as target stimuli, suggesting that the dorsal stream did not process the tool category specifically but rather the elongated shape common to many tools. To ascertain whether the shapes of other categories could influence the priming effect, we took advantage of the vegetable category, which has different shapes (i.e., elongated and stubby), as prime stimuli. With elongated vegetables as prime stimuli, we found significantly shortened RTs to subsequent tool targets, but no differences were seen when stubby vegetables were used as prime stimuli, indicating that elongated shapes of different forms shortened the time for judgment to tool targets. Our results suggest that the dorsal stream processes elongated shapes but does not process the tool category specifically.

Although Almeida et al. (2008) noted that dorsal stream computations influence object recognition processes for objects that can be manipulated, our results suggest that dorsal stream activation during priming may be due to shape attributes. Moreover, Sereno and Maunsell (1998) demonstrated shape selectivity in the primate lateral intraparietal area (LIP) in the dorsal stream. In addition, the equivalent of the LIP in humans is situated in the medial portion of the intraparietal sulcus (Grefkes and Fink, 2005), suggesting that this human brain area also might have shape selectivity. Vingerhoets et al. (2009) used fMRI to demonstrate that tool-like stimuli activated the dorsal portion of the parietal lobe, probably SPL. Thus, SPL may be activated because
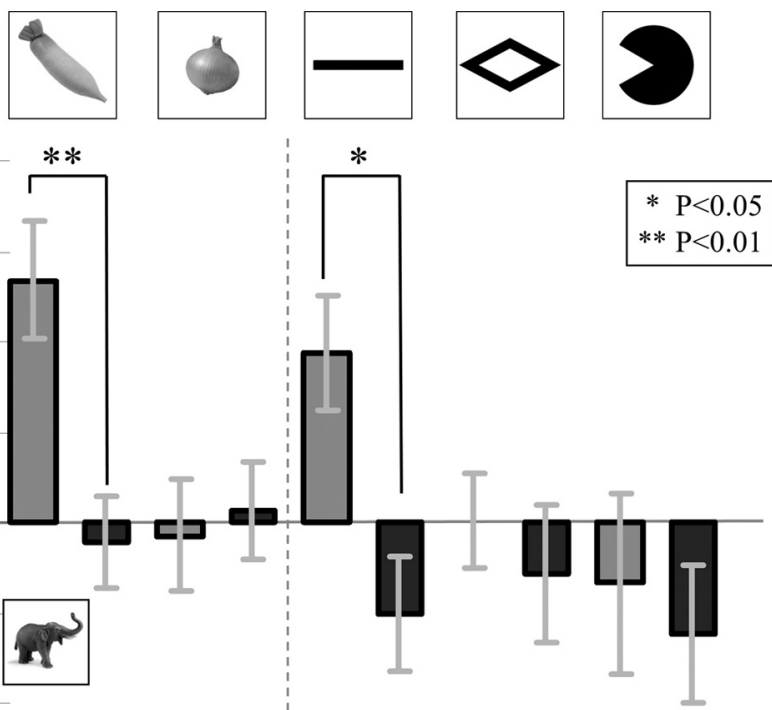

$* * \mathrm{P}<0.01$

Experiment 5
Experiment 4 riming effects to tool targets and Figure 4. Priming effect in Experiment 4 and 5. Light and dark gray bars represent mean priming effects to too ti
animal targets, respectively. Error bars indicate SEM. The pictures represent examples of the prime stimuli we used.

the objects contained a superficial elongated shape attribute in common with many tools. This "elongated" attribute of shape might be the essence of the term "manipulable" described by Almeida et al. (2008).

However, to make a definitive assertion that the dorsal stream processes information about elongated shapes, it is also necessary to verify that the results are specific to elongation. We cannot eliminate the possibility that these results are due to the orientation of the elongated shapes. Valyear et al. (2006) showed that areas in the right hemisphere dorsal stream show sensitivity to a change in object orientation. In our Experiment 3, two different angles of rectangles were used for prime stimuli, and it is possible that the directional attribute contributed to the priming effect. Almost all of the tool and elongated shape stimuli were presented at orientations $\pm 45^{\circ}$ from vertical. Because most, or possibly all, elongated shapes have direction, it is possible that the dorsal stream responded specifically to the directional attribute rather than the elongation. To clarify this, we conducted Experiment 5 using several kinds of rotated shapes: elongated stick figures, diamond shapes, and cut circles. Predictably, we found that significant differences were seen between tools and animals only when elongated stick figures were used as prime stimuli, and not when other oriented shapes were used. Considering those results, it is unlikely that the orientations of elongated shapes have a significant impact on the priming effect found in previous experiments.

There are some methodological open issues. Fang and $\mathrm{He}$ (2005) have raised questions regarding CFS. It is possible that a direct projection from the lateral geniculate nucleus to the middle temporal area or V5 processed the prime stimuli, or that CFS does not completely block cortical information from the suppressed eye. Unless these possibilities are eliminated, we cannot definitively establish the relationship between the dorsal stream and the elongated attribute of shape. Therefore, it will be necessary to use other methods, such as functional neuroimaging, to fully decipher dorsal stream processing.

However, our study using CFS indicates the possibility that the information which the dorsal stream processes is not "tools" as a category, but rather the elongated attribute of shape common to many tools. Milner and Goodale (2008) indicated that the 
dorsal stream is "vision for action" and differentiated it from "vision for perception," attributed to the ventral stream. Consistent with their suggestion, the information about elongated shapes processed in the dorsal stream may be used to specify not objects but how to act toward objects. It remains to be determined how the visual processing in the dorsal stream is transformed into action.

\section{References}

Almeida J, Mahon BZ, Nakayama K, Caramazza A (2008) Unconscious processing dissociates along categorical lines. Proc Natl Acad Sci U S A 105:15214-15218.

Almeida J, Mahon BZ, Caramazza A (2010) The role of the dorsal visual processing stream in tool identification. Psychol Sci 21:772-778.

Beauchamp MS, Lee KE, Haxby JV, Martin A (2002) Parallel visual motion processing streams for manipulable objects and human movements. Neuron 34:149-159.

Beauchamp MS, Lee KE, Haxby JV, Martin A (2003) fMRI responses to video and point-light displays of moving humans and manipulable objects. J Cogn Neurosci 15:991-1001.

Bracci S, Cavina-Pratesi C, Ietswaart M, Caramazza A, Peelen MV (2011) Closely overlapping responses to tools and hands in left lateral occipitotemporal cortex. J Neurophysiol doi:10.1152/jn.00619.2011

Cavina-Pratesi C, Kentridge RW, Heywood CA, Milner AD (2010) Separate channels for processing form, texture, and color; evidence from fMRI adaptation and visual object agnosia. Cereb Cortex 20:2319-2332.

Chao LL, Martin A (2000) Representation of manipulable man-made objects in the dorsal stream. Neuroimage 12:478-484.

Chao LL, Haxby JV, Martin A (1999) Attribute-based neural substrates in temporal cortex for perceiving and knowing about objects. Nat Neurosci 2:913-919.

Colby CL, Gattass R, Olson CR, Gross CG (1988) Topographical organization of cortical afferents to extrastriate visual area PO in the macaque: a dual tracer study. J Comp Neurol 269:392-413.

Fang F, He S (2005) Cortical responses to invisible objects in the human dorsal and ventral pathways. Nat Neurosci 8:1380-1385.
Foster KI, Foster JC (2003) DMDX: a windows display program with millisecond accuracy. Behav Res Meth Instrum Comput 35:116-124.

Goodale MA (2011) Transforming vision into action. Vision Res 51:1567-1587.

Goodale MA, Milner AD (1992) Separate visual pathways for perception and action. Trends Neurosci 15:20-25.

Goodale MA, Milner AD (2004) Sight unseen: an exploration of conscious and unconscious vision. Oxford: Oxford UP.

Goodale MA, Milner AD, Jakobson LS, Carey DP (1991) A neurological dissociation between perceiving objects and grasping them. Nature 349:154-156.

Grefkes C, Fink GR (2005) The functional organization of the intraparietal sulcus in humans and monkeys. J Anat 207:3-17.

Lin Z, He S (2009) Seeing the invisible: The scope and limits of unconscious processing in binocular rivalry. Prog Neurobiol 87:195-211.

Mahon BZ, Schwarzbach J, Caramazza A (2010) The representation of tools in left parietal cortex is independent of visual experience. Psychol Sci 21:764-771.

Milner AD, Goodale MA (2008) Two visual systems re-viewed. Neuropsychologia 46:774-785.

Rizzolatti G, Matelli M (2003) Two different streams form the dorsal visual system: anatomy and functions. Exp Brain Res 153:146-157.

Sereno AB, Maunsell JH (1998) Shape selectivity in primate lateral intraparietal cortex. Nature 395:500-503.

Tsuchiya N, Koch C (2005) Continuous flash suppression reduces negative afterimages. Nat Neurosci 8:1096-1101.

Valyear KF, Culham JC (2010) Observing learned object-specific functional grasps preferentially activates the ventral stream. J Cogn Neurosci 22:970-984.

Valyear KF, Culham JC, Sharif N, Westwood D, Goodale MA (2006) A double dissociation between sensitivity to changes in object identity and object orientation in the ventral and dorsal visual streams: A human fMRI study. Neuropsychologia 44:218-228.

Vingerhoets G, Acke F, Vandemaele P, Achten E (2009) Tool responsive regions in the posterior parietal cortex: effect of differences in motor goal and target object during imagined transitive movements. Neuroimage 47:1832-1843. 